\title{
Oostenrijk
}

\section{Länderbericht Österreich}

Univ.-Prof. Dr. Susanne Ferrari en Univ.-Ass. Dr. Valentina Köllich*

\section{Einführung}

Das österreichische Erbrecht ist erst kürzlich grundlegend reformiert worden. Das Erbrechtsänderungsgesetz 2015 (ErbRäG 2015), das mit 1. Jänner 2017 in Kraft getreten ist, stellt die umfangreichste Reform seit Bestehen des Allgemeinen Bürgerlichen Gesetzbuches (ABGB) 1811 dar. Kernbereiche betreffen insbesondere sprachliche und inhaltliche Anpassungen an die Bedürfnisse des 21. Jahrhunderts. So haben unter anderem das Testamentsrecht, das gesetzliche Erbrecht und das Pflichtteilsrecht Änderungen erfahren.

Im Folgenden werden drei Themen des österreichischen Erbrechts behandelt: die Stellung des überlebenden Ehegatten (Kapitel 2), die Stellung des überlebenden Lebensgefährten (Kapitel 3) und das Pflichtteilsrecht sowie pflichtteilsähnliche Rechte (Kapitel 4). Auch diese Bereiche wurden im Zuge der Reform (teilweise) neu geregelt und modernisiert, weshalb eine nähere Darstellung für den rechtsvergleichenden Diskurs vielversprechend erscheint.

Dr. Susanne Ferrari ist Universitätsprofessorin an der Karl-FranzensUniversität Graz. Dr. Valentina Köllich ist Universitätsassistentin an der Karl-Franzens-Universität Graz.

\section{Stellung des überlebenden Ehegatten}

\subsection{Gesetzliches Erbrecht}

Dem überlebenden Ehegatten bzw. eingetragenen Partner ${ }^{1}$ des Verstorbenen kommt in Österreich gemäß $\S 744$ ABGB ein gesetzliches Erbrecht zu. Dieses hat im Zuge des ErbRÄG 2015 - insbesondere wenn keine Nachkommen und Eltern des Verstorbenen vorhanden sind - abermals eine Stärkung erfahren. ${ }^{2}$ Das konkrete Ausmaß des Erbrechts hängt davon ab, welche miterbenden Verwandten vorhanden sind. Während dem Ehegatten neben den Verwandten der 1. Parentel (den Kindern des Verstorbenen und deren Nachkommen) ein Drittel der Verlassenschaft zusteht, erbt er neben den Eltern des Verstorbenen zwei Drittel der Verlassenschaft. Ist ein Elternteil vorverstorben, so fällt gemäß $\S 744$ Absatz 1 Satz 2 ABGB auch dessen Anteil dem Ehegatten zu. Somit werden auch Nachkommen der Eltern (Geschwister, Nichten, Neffen und so weiter) vom gesetzlichen Erbrecht ausgeschlossen, wenn der

1. Die erbrechtlichen Regelungen sind für Ehegatten und eingetragene Partner identisch. Die folgenden Ausführungen gelten für die Ehe und eingetragene Partnerschaft gleichermaßen, auch wenn letztere nicht ausdrücklich genannt wird. Das Rechtsinstitut der eingetragenen Partnerschaft steht in Österreich nur gleichgeschlechtlichen Personen zur Verfügung. Auf Grund einer Entscheidung des Verfassungsgerichtshofs im Dezember 2017 (VfGH G 258-259/2017-9 FamRZ 2018, 191 (Ferrari) $=\mathrm{EF}-Z$ 2018/31 (Höllwerth) $=\mathrm{iFamZ} \mathrm{2017/196}$ (Pesendorfer) $=\mathrm{JBI}$ 2018, 28 (Kerschner)) wird aber einerseits die eingetragene Partnerschaft ab dem 1.1.2019 auch für verschiedengeschlechtliche Paare geöffnet und andererseits die Ehe auch gleichgeschlechtlichen Paaren zugänglich gemacht. Beide Rechtsinstitute werden also sowohl gleichwie verschiedengeschlechtlichen Paaren zur Verfügung stehen.

2. Siehe zur stetigen Aufwertung des Ehegattenerbrechts seit der Urfassung: Scheuba in Kletečka/Schauer (Hrsg), Online-Kommentar zum ABGB - ABGB-ON ${ }^{1.03} \S 744$ ABGB Rz 3 (Stand 1.10.2017, rdb.at); Apathy/Musger in Koziol/P. Bydlinski/Bollenberger (Hrsg), Kurzkommentar zum $\mathrm{ABGB}^{5}$ (2017) § 744 ABGB Rz 1. 
Verstorbene zum Zeitpunkt des Todes verheiratet war. ${ }^{3}$ Im Fall, dass weder Personen aus der 1. noch aus der 2. Parentel vorhanden sind, wird der überlebende Ehegatte gemäß $§ 744$ Absatz 1 Satz 1 ABGB Alleinerbe und erhält die gesamte Verlassenschaft. Auf den ermittelten Erbteil des Ehegatten ist alles anzurechnen, was er durch einen Ehepakt oder Erbvertrag aus dem Vermögen des Verstorbenen erhält ( $\$ 744$ Abs. 2 ABGB). $\mathrm{Zu}$ beachten ist, dass $\S 744$ Abs. 1 ABGB nur den Ehegatten und nicht auch dessen Nachkommen zur gesetzlichen Erbfolge beruft. Folglich gibt es keine Repräsentation des vorverstorbenen oder erbunwürdigen Ehegatten. Dies steht jedoch einer Transmission (Vererben eines bereits angefallenen Erbrechts) nicht entgegen. ${ }^{4}$

Dem österreichischen Recht ist, anders als vielen anderen Rechtsordnungen, ein Güterausgleich, der im Fall des Todes eines Gatten dem Erbrecht vorgeht, fremd. ${ }^{5}$ Lediglich wenn in einem bereits anhängigen Scheidungsverfahren eine Vereinbarung über die ehelichen Ersparnisse und das eheliche Gebrauchsvermögen vorliegt, geht diese im Zweifel dem gesetzlichen Erbrecht vor. Siehe hierzu noch Kapitel 2.6.

\subsection{Vorausvermächtnis}

Dem überlebenden Ehegatten kommt gemäß $§ 745$ Abs. 1 ABGB zudem ein gesetzliches Vorausvermächtnis zu. Umfasst sind einerseits das Recht, in der Ehewohnung weiterzuwohnen, und andererseits die zum ehelichen Haushalt gehörenden beweglichen Sachen, soweit sie zu dessen Fortführung entsprechend den bisherigen Lebensverhältnissen erforderlich sind. ${ }^{6}$ Zweck des Vorausvermächtnisses ist es, dem überlebenden Ehegatten das gewohnte Lebensumfeld $\mathrm{zu}$ erhalten und $\mathrm{zu}$ sichern. ${ }^{7}$

Das Wohnrecht nach $\S 745$ Abs. 1 ABGB hat lediglich subsidiären Charakter. Es kommt somit nicht zur Anwendung, wenn der überlebende Gatte ein eigenes Recht an der Ehewohnung hat (beispielsweise als Eigentümer oder Mieter), oder ihm das Wohnrecht als Alleinerben, Vermächtnisnehmer, auf den Todesfall Beschenktem, als Eintrittsrechtberechtigtem in ein Mietverhältnis nach $\S 14$ Mietrechtsgesetz $(\mathrm{MRG})^{8}$ oder

3. Apathy, Erbrecht ${ }^{5}$ (2015) Rz 2/17.

4. Ferrari in Ferrari/Likar-Peer (Hrsg), Erbrecht: Ein Handbuch für die Praxis (2007) 70

5. Eccher, Erbrecht ${ }^{6}$ (2016) Rz 3/14. Siehe hierzu ausführlich Eccher, Ehegattenerbrecht und Güterausgleich, NZ 2011, 353.

6. Siehe $\S 745$ Abs. 1 ABGB. Vgl. die Ausführungen zu den Regelungen für Lebensgefährten in Kapitel 3.3.

7. Likar-Peer in Ferrari/Likar-Peer, Erbrecht 72; Welser/Zöchling-Jud, Grundriss des bürgerlichen Rechts II: Schuldrecht Allgemeiner Teil, Schuldrecht Besonderer Teil, Erbrecht ${ }^{14}$ (2015) Rz 1951; Apathy, Erbrecht $^{5}$ Rz 7/21; Apathy/Musger in $\mathrm{KBB}^{5} \S 745$ ABGB Rz 1.

8. Gem § 14 MRG ist unter anderem der Ehegatte des verstorbenen Mieters eintrittsberechtigt, wenn er schon bisher im gemeinsamen Haushalt gewohnt und ein dringendes Wohnbedürfnis hat.

9. § 14 WEG ist im Fall des gemeinsamen Wohnungseigentums des Verstorbenen und des überlebenden Gatten einschlägig. Letzterem wächst der Hälfteanteil des Verstorbenen an; dieser fällt nicht in die Verlassen-
Zuwachsberechtigtem nach $\S 14$ Wohnungseigentumsgesetz (WEG) ${ }^{9}$ zusteht. $^{10}$

Der überlebende Gatte ist im Rahmen des Wohnrechts berechtigt, die Wohnung unentgeltlich zu benützen. Es handelt sich um ein gesetzliches Dauerschuldverhältnis zwischen dem überlebenden Gatten und den Erben. Der überlebende Gatte hat hierbei die Betriebs- und Erhaltungskosten der Wohnung zu tragen. ${ }^{11}$ Das Wohnrecht endet mit dem Tod des berechtigten Gatten, aufgrund eines Verzichts oder durch Kündigung aus wichtigem Grund. ${ }^{12} \mathrm{Ob}$ auch die Wiederverheiratung zum Erlöschen führt, wird in der Lehre uneinheitlich beantwortet. ${ }^{13}$

Im Hinblick auf die beweglichen Haushaltsgegenstände, die zur Fortführung des Haushalts erforderlich sind, ist auf die tatsächliche Haushaltsführung und die Lebensverhältnisse der Gatten abzustellen. Nur jene Gegenstände, die der Haushaltsführung gedient haben, sind davon umfasst und fallen sodann in das Eigentum des überlebenden Gatten. Beispielhaft seien Einrichtungsgegenstände, Geschirr, Bilder, Teppiche oder Fernsehgeräte genannt. $^{14}$

Das Vorausvermächtnis steht unabhängig davon $\mathrm{zu}, \mathrm{ob}$ der Gatte testamentarischer oder gesetzlicher Erbe oder lediglich Pflichtteilsberechtigter ist. Weiters ist die Höhe der Erb- oder Pflichtteilsquote unerheblich für das Vorausvermächtnis. ${ }^{15} \mathrm{Da}$ das Vorausvermächtnis pflichtteilsähnlichen Charakter hat, kann es nur bei Vorliegen eines Enterbungsgrundes entzogen werden. ${ }^{16}$ Der überlebende Gatte kann jedoch zu Lebzeiten seines Gatten auf das Vorausvermächtnis verzichten oder dies nach dem Tod seines Gatten ausschlagen. ${ }^{17}$

Nach herrschender Ansicht ${ }^{18}$ kommt dem überlebenden Ehegatten das gesetzliche Vorausvermächtnis zusätzlich zum gesetzlichen Erbteil zu. Dies ergibt sich aus einem Umkehrschluss aus $§ 744$ Absatz 2 ABGB, welcher normiert, dass sich der Ehegatte alles auf den Erbteil anzurechnen hat, was er durch einen Ehepakt oder Erbvertrag aus dem Vermögen des Verstorbenen erhält. Dem Verstorbenen steht es frei, ein Hineinvermächtnis und somit die Anrechnung auf den gesetzlichen Erbteil

schaft. Der Überlebende hat jedoch grundsätzlich einen Übernahmepreis in Höhe des halben Verkehrswerts des Mindestanteils an die Verlassenschaft zu bezahlen, sofern der Verstorbene nichts Anderes angeordnet hat.

10. Apathy, Erbrecht ${ }^{5} \mathrm{Rz}$ 7/23; Eccher, Erbrecht ${ }^{6}$ Rz 9/33; Apathy/Musger in $\mathrm{KBB}^{5} \S 745 \mathrm{ABGB} \mathrm{Rz} 4$; Nemeth in Schwimann/Neumayr (Hrsg), ABGB-Taschenkommentar ${ }^{4}$ (2017) § 745 ABGB Rz 10.

11. Apathy, Erbrecht ${ }^{5} \mathrm{Rz} 7 / 24$; Apathy/Musger in $\mathrm{KBB}^{5} \S 745 \mathrm{ABGB}$ Rz 6 .

12. Likar-Peer in Ferrari/Likar-Peer, Erbrecht 91; Nemeth in Schwimann/ Neumayr, ABGB Takomm ${ }^{4} \S 745$ ABGB Rz 14

13. Siehe hierzu Likar-Peer in Ferrari/Likar-Peer, Erbrecht 93; Nemeth in Schwimann/Neumayr, ABGB Takomm ${ }^{4} \S 745$ ABGB Rz 14.

14. Eccher, Erbrecht ${ }^{6}$ Rz 9/36; Apathy/Musger in $\mathrm{KBB}^{5} \S 745 \mathrm{ABGB}$ Rz 3.

15. Likar-Peer in Ferrari/Likar-Peer, Erbrecht 72.

16. Likar-Peer in Ferrari/Likar-Peer, Erbrecht 78 f.; Apathy, Erbrecht ${ }^{5} \mathrm{Rz}$ 7/21; Apathy/Musger in $\mathrm{KBB}^{5} \S 745$ ABGB Rz 1.

17. Likar-Peer in Ferrari/Likar-Peer, Erbrecht 79.

18. Siehe hierzu die Nachweise bei Likar-Peer in Ferrari/Likar-Peer, Erbrecht 72. 
anzuordnen. Dem überlebenden Gatten muss in einem solchen Fall jedoch zumindest der Pflichtteil zukommen. ${ }^{19}$

Wird der überlebende Ehegatte demgegenüber testamentarischer Miterbe, so hängt es von der Auslegung der letztwilligen Verfügung $a b$, ob eine Einrechnung in den testamentarischen Erbteil erfolgen soll. Im Zweifel gebührt das Vorausvermächtnis aber neben dem testamentarischen Erbteil. Anderes gilt, wenn der Ehegatte lediglich den Pflichtteil erhält. In diesen ist das Vorausvermächtnis einzurechnen, es sei denn, der Verstorbene hat die Einrechnung erlassen. ${ }^{20}$

\subsection{Unterhalt}

Der nach § 94 ABGB während der Ehe zustehende Unterhaltsanspruch erlischt mit dem Tod des Unterhalspflichtigen. ${ }^{21}$ Dem überlebenden Ehegatten gebührt gemäß $§ 747$ ABGB aber ein Unterhaltsanspruch gegen die Erben, solange er nicht erneut heiratet. Dieser, in Form einer Geldrente zu leistende Unterhalt, orientiert sich am ehelichen Unterhalt gemäß § 94 ABGB, ist aber mit dem Wert der Verlassenschaft begrenzt. ${ }^{22}$ Der Anspruch steht nicht zu, wenn der überlebende Ehegatte erbunwürdig ist oder enterbt wurde. ${ }^{23}$

Anders als beim Vorausvermächtnis hat sich der überlebende Ehegatte gemäß $\S 747$ ABGB alles anrechnen zu lassen, was er nach dem Verstorbenen durch vertragliche oder letztwillige Zuwendung, als gesetzlichen Erbteil, als Pflichtteil und durch öffentlich-rechtliche oder privatrechtliche Leistungen erhält. Des Weiteren ist auch Vermögen sowie Erträgnisse einer tatsächlich ausgeübten oder einer dem überlebenden Gatten zumutbaren Erwerbstätigkeit zu berücksichtigen.

\subsection{Erbvertrag}

In der Praxis wenig Bedeutung hat der Erbvertrag zwischen dem Verstorbenen und dem Ehegatten gemäß $\S \S 602,1249 \mathrm{ff}$ ABGB. Hierbei handelt es sich um ein zweiseitiges Rechtsgeschäft, bei dem ein Gatte den anderen oder beide sich gegenseitig zu Erben einsetzen. ${ }^{24}$ Der Erbvertrag stellt den stärksten Berufungsgrund dar, sodass der erbvertraglich eingesetzte Erbe allen anderen Erben vorgeht. ${ }^{25} \mathrm{Zu}$ beachten ist, dass sich der Erbvertrag gemäß $§ 1253$ ABGB höchstens auf drei Viertel des Vermögens beziehen darf und zudem einerseits die Formvorschriften eines Notariatsaktes und andererseits jene eines schriftlichen Testaments erfüllen muss. ${ }^{26}$
Im Fall der Scheidung wird gemäß $§ 1266$ ABGB nach dem Verschulden der Gatten differenziert: Bei Vorliegen gleichteiligen oder keines Verschuldens sowie bei einvernehmlicher Scheidung erlischt der Erbvertrag für beide Teile. Wurde demgegenüber im Scheidungsurteil das alleinige oder überwiegende Verschulden des verstorbenen Gatten ausgesprochen, so bleibt der Erbvertrag zugunsten des überlebenden, minderschuldigen bzw. schuldlosen Teiles aufrecht.

\subsection{Pflichtteil}

Der Ehegatte des Verstorbenen ist gemäß $§ 757$ ABGB pflichtteilsberechtigt. Siehe hierzu ausführlich Kapitel 4.

\subsection{Stellung des Ehegatten bei aufgelöster Ehe und anhängigem Auflösungsverfahren}

Nach rechtskräftiger Auflösung der Ehe durch Scheidung, Aufhebung oder Nichtigerklärung kommt dem geschiedenen Gatten weder das gesetzliche Erbrecht nach $\$ 744$ Absatz 1 ABGB noch das gesetzliche Vorausvermächtnis nach $\S 745$ Absatz 1 ABGB zu. Auch der Unterhaltsanspruch nach $\S 747$ ABGB gebührt im Fall einer zum Todeszeitpunkt bereits aufgelösten Ehe nicht. ${ }^{27}$ Denkbar wäre aber das Vorliegen eines nachehelichen Unterhaltsanspruchs, der gemäß $§ 78$ Ehegesetz (EheG) mit dem Tod des Verpflichteten auf die Erben übergeht. ${ }^{28}$ Lediglich Rechte aus einem Erbvertrag könnten bei einer zum Zeitpunkt des Erbfalls bereits aufgelösten Ehe in jenem Fall zustehen, in dem im Scheidungsurteil das alleinige oder überwiegende Verschulden des verstorbenen, geschiedenen Gatten ausgesprochen wurde. ${ }^{29}$

Die zeitliche Grenze bildet somit die Rechtskraft der Auflösung der Ehe. Eine Ausnahme von dieser Grundregel findet sich in $\$ 746$ Absatz 2 ABGB, der durch das ErbRÄG 2015 neu eingeführt wurde. Das gesetzliche Vorausvermächtnis und das Erbrecht stehen dem überlebenden Gatten - obwohl die Ehe noch aufrecht ist auch dann nicht zu, wenn zum Zeitpunkt des Erbfalls ein Verfahren über die Auflösung der Ehe anhängig ist und in diesem eine Vereinbarung über die Aufteilung des ehelichen Gebrauchsvermögens und der Ersparnisse für den Fall der Rechtskraft der Auflösungsentscheidung vorliegt. Diese gilt nämlich im Zweifel auch für die Auflösung der Ehe durch den Tod eines Ehegatten. Da es sich lediglich um eine gesetzliche Vermutung handelt, können die Ehegatten vereinbaren, dass es im
19. Apathy, Erbrecht ${ }^{5} \mathrm{Rz} 7 / 21$.

20. Likar-Peer in Ferrari/Likar-Peer, Erbrecht 73

21. Hopf/Kathrein, Kommentar zum Eherecht ${ }^{3}$ (2014) § 94 ABGB Rz 46; Schwimann/Ferrari in Schwimann/Neumayr, ABGB Takomm ${ }^{4} \S 94$ ABGB Rz 49.

22. Weiß/Ferrari in Ferrari/Likar-Peer, Erbrecht $33 \mathrm{f}$.

23. Apathy/Musger in $\mathrm{KBB}^{5} \S 747 \mathrm{ABGB}$ Rz 2.

24. Welser/Zöchling-Jud, Bürgerliches Recht II ${ }^{14}$ Rz 1983.

25. Apathy, Erbrecht ${ }^{5} \mathrm{Rz} 8 / 1$.

6. § 1249 S 2 ABGB; § 1 Abs. 1 lit a NAktG.
27. Dem Gesetzestext zufolge gebührt der Unterhaltsanspruch nach § 747 ABGB auch dann nicht, wenn zum Zeitpunkt des Erbfalls ein Verfahren über die Auflösung der Ehe anhängig ist und in diesem eine Vereinbarung über die Aufteilung des ehelichen Gebrauchsvermögens und der Ersparnisse für den Fall der Rechtskraft der Auflösungsentscheidung vorliegt. Hierbei handelt es sich wohl um ein Redaktionsversehen, weshalb eine teleologische Reduktion geboten erscheint. Vgl. Bittner/Hawel in Kletečka/Schauer, ABGB-ON ${ }^{1.03} \S 747$ ABGB Rz 2.

28. Dies gilt gem $\S 78$ Abs. 3 EheG nicht für den Unterhaltsbeitrag nach $\S 68$ EheG, der bei Scheidung aus Verschulden mit Ausspruch des gleichteiligen Verschuldens der Gatten gebührt.

29. Siehe hierzu bereits Kapitel 2.4, sowie § 1266 ABGB. 
Fall des Todes beim gesetzlichen Erbrecht der Ehegatten bleibt. ${ }^{30}$

Vereinbarungen über die Aufteilung des ehelichen Gebrauchsvermögens und der ehelichen Ersparnisse können beispielsweise im Zuge einer einvernehmlichen Scheidung gemäß § 55a Absatz 2 EheG oder im Rahmen eines Eheauflösungsverfahrens gemäß § 97 Absatz 5 EheG getroffen werden. ${ }^{31}$ Fraglich ist, ob auch sogenannte Vorwegvereinbarungen gemäß § 97 Absatz 1 EheG, die im Voraus die Aufteilung des ehelichen Gebrauchsvermögens und der ehelichen Ersparnisse regeln, umfasst sind. ${ }^{32}$ Probleme bereitet in diesem Zusammenhang der Umstand, dass Vorwegvereinbarungen im Aufteilungsverfahren einer gerichtlichen Kontrolle unterliegen. Im Fall des Todes eines der Gatten findet jedoch kein Aufteilungsverfahren mehr statt. Teile der Lehre treten deshalb zutreffender Weise auch im Rahmen des $§ 746$ Absatz 2 ABGB für eine gerichtliche Kontrolle nach $\S 97$ Absatz 2 bis 4 EheG ein. ${ }^{33}$

Auch eine testamentarische Begünstigung des Ehegatten wird gemäß $§ 725$ ABGB mit der Scheidung aufgehoben, außer der Verstorbene hat etwas Anderes angeordnet. Im Zweifel wird die den Ehegatten betreffende letztwillige Anordnung auch dann aufgehoben, wenn zu Lebzeiten des Verstorbenen ein Verfahren zur Auflösung der Ehe eingeleitet wurde.

\section{Stellung des überlebenden Lebensgefährten}

\subsection{Allgemeines}

Auch die Stellung des Lebensgefährten wurde durch das ErbRÄG 2015 - wie jene des Ehegatten - gestärkt. Zuvor hatte dieser keine erbrechtlichen Ansprüche. ${ }^{34}$ Nunmehr gebührt dem Lebensgefährten unter bestimmten - wenngleich sehr strengen - Voraussetzungen ein gesetzliches Erbrecht sowie ein gesetzliches Vorausvermächtnis.

\subsection{Gesetzliches Erbrecht}

Dem überlebenden Lebensgefährten des Verstorbenen gebührt gemäß $§ 748$ ABGB ein außerordentliches Erb-

30. Apathy, Erbrecht ${ }^{5}$ Rz 2/16.

31. Deixler-Hübner, Erbrechtliche Absicherung des Ehegatten, eingetragenen Partners und Lebensgefährten, in Barth/Pesendorfer (Hrsg), Praxishandbuch des neuen Erbrechts (2016) 39 (44); Eccher, Erbrecht ${ }^{6}$ Rz $3 / 14$.

32. Siehe zu den unterschiedlichsten Ansichten bspw Deixler-Hübner in Barth/Pesendorfer $44 \mathrm{f}$.; Scheuba in Kletečka/Schauer, ABGB-ON ${ }^{1.03}$ $\S 746$ ABGB Rz 7; Apathy/Musger in $\mathrm{KBB}^{5} \S 746$ ABGB Rz 4.

33. Fischer-Czermak/Gitschthaler/A. Tschugguel, Tod des Ehegatten/eingetragenen Partners während eines Auflösungsverfahrens, EF-Z 2017, 148 (151 f.). AA Fischer-Czermak/Gitschthaler/A. Tschugguel, EF-Z 2017, 150 f.; Deixler-Hübner in Barth/Pesendorfer 44 f.; Apathy/Musger in $\mathrm{KBB}^{5}$ § 746 ABGB Rz 4; Apathy/Musger in $\mathrm{KBB}^{5}$ § 746 ABGB Rz 4.

34. Scheuba in Kletečka/Schauer, ABGB-ON ${ }^{1.03} \S 746$ ABGB Rz 1. Dieser wurde nur in Ausnahmefällen berücksichtigt, siehe bspw § 14 Abs. 3 MRG.

recht, das entgegen des verwendeten Terminus ein gesetzliches Erbrecht darstellt. ${ }^{35}$ Dies steht dem Lebensgefährten jedoch nur dann $\mathrm{zu}$, wenn weder gesetzliche noch vertragliche oder testamentarische Erben die Verlassenschaft erwerben. Das Erbrecht des Lebensgefährten geht somit lediglich dem außerordentlichen Erbrecht der Vermächtnisnehmer und dem Aneignungsrecht des Bundes vor. ${ }^{36}$ Aus diesem Grund ist die Bedeutung in der Praxis wohl eher gering.

$\S 748$ Absatz 1 ABGB setzt überdies voraus, dass der Lebensgefährte und der Verstorbene zumindest in den letzten drei Jahren vor dem Tod des Verstobenen im gemeinsamen Haushalt gelebt haben. Dieses Erfordernis kann jedoch gemäß $§ 748$ Absatz 2 ABGB aus erheblichen Gründen gesundheitlicher oder beruflicher Art entfallen, wenn eine für Lebensgefährten typische besondere Verbundenheit bestand. Als Beispiel kann genannt werden, dass ein Lebensgefährte pflegebedürftig ist und deshalb in einem Heim lebt. Besteht die Verbundenheit fort, beispielsweise durch Besuche und gegenseitige Anteilnahme, so gebührt dem überlebenden Lebensgefährten das Erbrecht nach $\$ 748$ ABGB. ${ }^{37}$

\subsection{Gesetzliches Vorausvermächtnis}

Auch dem Lebensgefährten gebührt ein gesetzliches Vorausvermächtnis gemäß $§ 745$ Absatz 2 ABGB. Dieses umfasst - wie beim überlebenden Ehegatten - einerseits das Recht, weiter in der Wohnung zu wohnen, und andererseits die zum ehelichen Haushalt gehörenden beweglichen Sachen, soweit sie zu dessen Fortführung entsprechend den bisherigen Lebensverhältnissen erforderlich sind.

Vorausgesetzt wird gemäß $§ 745$ Absatz 2 Satz 1 ABGB, dass der Überlebende mit dem Lebensgefährten zumindest in den letzten drei Jahren vor dem Tod des Verstorbenen im gemeinsamen Haushalt gelebt hat und der Verstorbene im Zeitpunkt seines Todes weder verheiratet war noch in einer eingetragenen Partnerschaft gelebt hat. Demgegenüber ist es gleichgültig, ob der Überlebende zum Todeszeitpunkt seines Lebensgefährten noch verheiratet ist oder einen eingetragenen Partner hat. ${ }^{38}$ Anders als das Vorausvermächtnis des Ehegatten ist jenes des überlebenden Lebensgefährten gemäß $§ 745$ Absatz 2 Satz 2 ABGB auf ein Jahr nach dem Tod des Verstorbenen befristet.

Uneinheitlich beantwortet wird die Frage, ob die Aufhebung der Haushaltsgemeinschaft aus 'erheblichen Gründen' - wie auch im Rahmen des außerordentlichen Erbrechts gemäß $§ 748$ Absatz 2 ABGB - zulässig ist. Dies wird wohl insbesondere aufgrund historischer Argumente zu bejahen sein. Der überlebende Lebensge-

35. er, $A B G B-O N^{1.03} \S 748$ ABGB Rz 2

36. Apathy, Erbrecht ${ }^{5} \mathrm{Rz} 2 / 19$; Apathy/Musger in $K B B^{5}$ § $748 \mathrm{ABGB}$ Rz 1.

37. Apathy, Erbrecht ${ }^{5}$ Rz 2/20.

38. Nemeth in Schwimann/Neumayr, ABGB Takomm ${ }^{4} \S 745$ ABGB Rz 18 
fährte muss jedoch jedenfalls zum Zeitpunkt des Todes in der Wohnung gelebt haben. ${ }^{39}$

Wie bereits erwähnt, hat auch der Lebensgefährte nach $\S 745$ Absatz 1 und Absatz 2 ABGB das Recht auf die zum Haushalt gehörenden beweglichen Sachen, soweit sie zu dessen Fortführung entsprechend den bisherigen Lebensverhältnissen erforderlich sind. Dem Lebensgefährten wird hierbei nach Ansicht der herrschenden Lehre jedoch - anders als dem Ehegatten - kein Eigentumsrecht eingeräumt. Teilweise wird vertreten, dass der Lebensgefährte die Stellung eines Vorvermächtnisnehmers hat, der fruchtgenussberechtigt ist. ${ }^{40}$ Andere sprechen ihm lediglich ein Gebrauchsrecht an den Haushaltsgegenständen $\mathrm{zu}^{41}$

\section{Pflichtteilsrecht und pflichtteilsähnliche Rechte}

\subsection{Allgemeines}

Das österreichische Pflichtteilsrecht schränkt die Testierfreiheit erheblich ein, da grundsätzlich gewissen Familienangehörigen des Verstorbenen ein Mindestanteil an der Verlassenschaft - in Form eines Geldpflichtteils - zukommen muss, auch wenn der Verstorbene sein gesamtes Vermögen testamentarisch oder erbvertraglich anderen Personen zukommen lassen will. Aus diesem Grund hat die Regelung des Pflichtteilsrechts auch gesellschaftspolitische Brisanz. ${ }^{42}$ Bereits im Vorfeld des ErbR ÄG 2015 wurde eine Reform des Pflichtteilsrechts gefordert. Teile der Lehre sprachen sich sogar für eine gänzliche Abschaffung des Pflichtteils aus. ${ }^{43}$ Das ErbRÄG 2015 hat dieser Forderung nicht Rechnung getragen und das Pflichtteilsrecht beibehalten. Es ist jedoch in manchen Bereichen zu grundlegenden Änderungen gekommen, und die Testierfreiheit hat eine Stärkung erfahren. ${ }^{44}$

\subsection{Kreis der pflichtteilsberechtigten Personen}

$\mathrm{Zu}$ den abstrakt pflichtteilsberechtigten Personen zählen gemäß $§ 757$ ABGB die Nachkommen des Verstorbenen (Kinder, Enkel, Urenkel und so weiter) sowie der Ehegatte bzw. eingetragene Partner; nicht jedoch der

39. Siehe bspw ErläutRV 688 BlgNR 25. GP 21. Ähnlich auch Deixler-Hübner in Barth/Pesendorfer 49; Apathy, Erbrecht ${ }^{5} \mathrm{Rz}$ 7/22; Apathy/ Musger in $\mathrm{KBB}^{5} \S 745 \mathrm{ABGB} \mathrm{Rz}$ 11. AA Scheuba in Kletečka/Schauer, ABGB-ON ${ }^{1.03} \S 745$ ABGB Rz 15.

40. So bspw Barth/Pesendorfer, Erbrechtsreform 2015 (2015) 81; Verweijen, ErbRÄG 2015 - zu den Rechten des Lebensgefährten, ÖJZ 2016, 853 (854)

41. Siehe auch Beclin, Nichteheliche Lebensgemeinschaften, in DeixlerHübner (Hrsg), Handbuch Familienrecht 115 (163); Schauer, Familie und Erbrecht, in Deixler-Hübner (Hrsg), Handbuch Familienrecht 689 (706); Deixler-Hübner in Barth/Pesendorfer 49; Apathy, Erbrecht ${ }^{5} \mathrm{Rz}$ 7/30; Zankl, Erbrecht - Lehr- und Praxishandbuch ${ }^{8}$ (2017) 44

42. Eccher, Erbrecht ${ }^{6}$ Rz $11 / 1$.

43. Siehe hierzu die zahlreichen Nachweise bei Kalss/Cach, Das neue Pflichtteilsrecht, Aufsichtsrat aktuell 2015, 5 FN 2.

44. Siehe bspw Barth, Das neue Pflichtteilsrecht, iFamZ 2015, 237; Eliskases, ErbRÄG 2015 - Das neue Pflichtteilsrecht, Zak 2016, 344; Mondel, Der nicht mehr so 'freie' Pflichtteil, iFamZ 2016, $180 \mathrm{f}$.
Lebensgefährte. Vor der Reform waren auch noch die Eltern, Großeltern und Urgroßeltern des Verstorbenen pflichtteilsberechtigt. ${ }^{45}$ Diese Regelung wurde unter anderem mit der Begründung, dass wohl meist Kinder nicht vor ihren Eltern bzw. Groß- oder sogar Urgroßeltern sterben, als nicht mehr zeitgemäß angesehen und aufgehoben. ${ }^{46}$

Dem genannten - abstrakt pflichtteilsberechtigten Personenkreis kommt der Pflichtteil jedoch gemäß $§ 758$ ABGB nur in jenem Fall zu, in dem sie auch konkret pflichtteilsberechtigt sind. Dies liegt vor, wenn ihnen im konkreten Fall ohne letztwillige Verfügung ein gesetzlicher Erbteil zustehen würde und sie infolgedessen nicht vorverstoben sind, enterbt wurden oder auf ihren Pflichtteil verzichtet haben. ${ }^{47}$

\subsection{Pflichtteilsberechnung}

Die Berechnung der Höhe des Pflichtteils ergibt sich aus $\S 759$ ABGB. Demnach gebührt als Pflichtteil die Hälfte dessen, was nach gesetzlicher Erbfolge zustünde. Im Vorfeld ist somit zu klären, wie viel dem Pflichtteilsberechtigten im konkreten Sachverhalt, ohne Vorliegen eines Testaments oder eines Erbvertrages bei gesetzlicher Erbfolge zugekommen wäre. Im Folgenden werden deshalb in einem ersten Schritt die Grundzüge der Regelungen zur Berechnung der gesetzlichen Erbfolge dargelegt, um die Pflichtteilsberechnung anschließend mit Beispielen besser veranschaulichen zu können.

Gemäß $§ 732$ ABGB fällt den Kindern des Verstorbenen die gesamte Verlassenschaft zu gleichen Teilen zu, wenn der Verstorbene keinen Ehegatten hinterlässt. Ist ein Kind vor dem Verstorbenen gestorben und hinterlässt dieses Nachkommen (somit Enkel des Verstorbenen), so fällt den Enkeln gemäß $§ 733$ ABGB der Erbteil $\mathrm{zu}$ gleichen Teilen $\mathrm{zu}$; sie repräsentieren somit ihren Elternteil.

Hinterlässt der Verstorbene einen Ehegatten, so gebührt diesem gemäß $§ 744$ Absatz 1 ABGB neben den Verwandten der 1. Parentel (den Kindern des Verstorbenen und deren Nachkommen) ein Drittel der Verlassenschaft, während ihm neben den Eltern des Verstorbenen zwei Drittel der Verlassenschaft zukommen. Ist ein Elternteil vorverstorben, so fällt auch dessen Anteil dem Ehegatten $\mathrm{zu}^{48}$

\section{Beispiel 1}

Der Verstorbene $\mathrm{V}$ hinterlässt neben seinen 3 Kindern K1, K2 und K3 seinen Ehegatten E. Nach gesetzlicher Erbfolge kommt den Kindern gemäß $\S 732$ ABGB die gesamte Verlassenschaft zu. Dem Ehegatten gebührt neben den Kindern als 1. Parentel gemäß $\$ 744$ ABGB 1/3 der Verlassenschaft. Den Kindern würden nach gesetzlicher Erbfolge 2/3 zustehen, pro Kind somit 2/9. Da der Pflichtteil nur

\footnotetext{
45. Siehe hierzu § $762 \mathrm{aF}$.

46. Siehe hierzu die Begründung in den ErläutRV 688 BlgNR 25. GP 24

47. Apathy, Erbrecht ${ }^{5}$ Rz 4/5.

48. Siehe dazu schon oben Kapitel 2.1.
} 
die Hälfte des gesetzlichen Erbteils ausmacht, gebührt dem Gatten 1/6 und jedem Kind 1/9. Dem bzw. den Erben verbleibt daher wertmäßig nur die halbe Verlassenschaft.

\section{Beispiel 2}

Der Verstorbene hinterlässt seinen Ehegatten E, zwei Kinder K1 und K3 sowie die Enkeln X1, X2 und X3 als Kinder des vorverstorbenen Kindes K2. Der Ehegatte erhält neben den Kindern wiederum 1/3 der Verlassenschaft als gesetzlichen Erbteil. Die Kinder bekommen gemeinsam den Rest in Höhe von 2/3, somit $2 / 9$ pro Kind. Da K2 jedoch vorverstorben ist, fällt sein Teil an seine Kinder, die Enkel X1, X2 und X3. Im Ergebnis erhält jeder Enkel 2/27 als gesetzlichen Erbteil. Der Pflichtteil beträgt wiederum die Hälfte von den soeben dargelegten Quoten. Der Ehegatte E1 erhält somit $1 / 6$, die Kinder $\mathrm{K} 1$ und $\mathrm{K} 3$ bekommen 1/9 und den Enkeln X1, X2 und X3 gebührt 1/27 als Pflichtteil. Wiederum verbleibt den Erben nur der halbe Verlassenschaftswert.

Die Bemessungsgrundlage des Pflichtteils richtet sich gemäß § 779 ABGB nach der reinen Verlassenschaft. Somit sind von den Verlassenschaftsaktiven die Schulden abzuziehen. Hierzu zählen neben den Erblasserschulden auch Begräbniskosten und Kosten für die Verlassenschaftsabhandlung (Erbgangsschulden), nicht jedoch Vermächtnisse. Die Bewertung der Verlassenschaft erfolgt nach dem gemeinen Wert (Verkehrswert) zum Zeitpunkt des Erbfalls. ${ }^{49}$

\subsection{Ausschluss vom Pflichtteil}

\subsubsection{Enterbung}

Wie bereits erwähnt, hat das Pflichtteilsrecht grundsätzlich zwingenden Charakter. Den konkret pflichtteilsberechtigten Personen hat der Pflichtteil jedenfalls zuzukommen. Die einzige Möglichkeit, den gesamten Pflichtteil zu entziehen, stellt gemäß $§ 769$ ABGB die Enterbung dar. Hierfür müssen jedoch bestimmte Voraussetzungen gegeben sein. Einerseits hat ein in $\S 770$ ABGB taxativ aufgezählter Enterbungsgrund vorzuliegen, der kausal für die Enterbung ist, und andererseits muss die Enterbung ausdrücklich in einer letztwilligen Verfügung oder stillschweigend durch Übergehung in einer letztwilligen Verfügung erfolgen. ${ }^{50}$ Die Beweislast für das Vorliegen eines Enterbungsgrundes trifft den Pflichtteilsschuldner. ${ }^{51}$

§770 ABGB normiert folgende Enterbungsgründe:

1. Das Begehen einer gerichtlich strafbaren Handlung gegen den Verstorbenen, die nur vorsätzlich begangen werden kann und mit mehr als einjähriger Freiheitsstrafe bedroht ist.

2. Das Begehen einer gerichtlich strafbaren Handlung gegenüber dem Verstorbenen nahestehenden Perso- nen (beispielsweise Ehegatte, Lebensgefährte, Verwandte in gerader Linie und so weiter), die nur vorsätzlich begangen werden kann und mit einer mehr als einjährigen Freiheitsstrafe bedroht ist.

3. Die absichtliche Vereitelung oder versuchte Vereitelung der Verwirklichung des wahren letzten Willens des Verstorbenen.

4. Das Zufügen schweren seelischen Leids gegenüber dem Verstorbenen.

5. Die gröbliche Vernachlässigung familienrechtlicher Pflichten gegenüber dem Verstorbenen.

6. Die Verurteilung zu einer lebenslangen oder zwanzigjährigen Freiheitsstrafe wegen einer oder mehrerer mit Vorsatz begangenen strafbaren Handlungen.

Ein weiterer Enterbungsgrund findet sich in $\S 771$ ABGB. Demnach kann aufgrund der Verschuldung oder eines verschwenderischen Lebensstils eines Pflichtteilsberechtigten, der ihm gebührende Pflichtteil zugunsten seiner Kinder entzogen werden.

Gemäß $§ 773$ ABGB besteht jedoch auch die Möglichkeit, die Enterbung zu widerrufen. Dies kann ausdrücklich durch Widerruf der Enterbung oder stillschweigend durch Bedenkung des vorher Enterbten erfolgen. Konnte der Verstorbene aufgrund seiner fehlenden Testierfähigkeit die Enterbung nicht mehr widerrufen, so ist die Enterbung dann unwirksam, wenn der Verstorbene zu erkennen gegeben hat, dass er dem Enterbten verziehen hat.

\subsubsection{Pflichtteilsminderung}

Die Pflichtteilsminderung gemäß $§ 776$ ABGB bietet dem Verfügenden die Möglichkeit, den Pflichtteil auf die Hälfte zu reduzieren. Auch dies muss, wie die Enterbung, in einer letztwilligen Verfügung erfolgen. Für die Pflichtteilsminderung wird vorausgesetzt, dass der Verstorbene und der Pflichtteilsberechtigte entweder zu keinem Zeitpunkt oder über einen längeren Zeitraum vor dem Tod des Verfügenden nicht in einem Naheverhältnis standen, wie es zwischen solchen Familienangehörigen üblich besteht. Ein längerer Zeitraum wird ungefähr ab 20 Jahren des fehlenden Kontakts angenommen. ${ }^{52}$ Ein fehlendes Naheverhältnis liegt dann vor, wenn eine objektive, durch fehlenden üblichen Kontakt entstandene Entfremdung gegeben ist; ein bloßes Gefühl der Entfremdung genügt demgegenüber nicht. ${ }^{53}$ Ausgenommen sind gemäß § 766 Absatz 2 ABGB jedoch jene Fälle, in denen der Verstorbene den Kontakt grundlos gemieden oder selbst Anlass für den fehlenden Kontakt gegeben hat.

\subsection{Anfall und Fälligkeit}

Der Pflichtteilsanspruch als schuldrechtlicher Anspruch des Pflichtteilsberechtigten, der nicht oder nicht ausreichend in einer letztwilligen Verfügung bedacht wurde, richtet sich gemäß $§ 764$ ABGB zunächst gegen die Ver- 
lassenschaft und ab Einantwortung ${ }^{54}$ gegen die Erben. Er ist, wie bereits erwähnt, in Geld zu leisten, wenn der Verstorbene ihn nicht durch eine Zuwendung von Todes wegen oder durch eine Schenkung unter Lebenden schon gedeckt hat. ${ }^{55}$

Gemäß $§ 765$ Absatz 1 ABGB erwirbt der Pflichtteilsberechtigte seinen Pflichtteilsanspruch mit dem Tod des Verstorbenen. Die Möglichkeit zur Geltendmachung des Geldpflichtteilanspruchs besteht gemäß $\S 765$ Absatz 2 ABGB jedoch erst ein Jahr nach dem Erbfall. Bis zur Erfüllung des Geldpflichtteils stehen dem Pflichtteilsberechtigten die gesetzlichen Zinsen zu. ${ }^{56}$

\subsection{Stundung}

Seit dem ErbRÄG 2015 besteht die Möglichkeit zur Stundung des Pflichtteils. Hintergedanke ist, dass der Pflichtteilsschuldner durch die sofortige Verpflichtung zur Pflichtteilszahlung nicht in seiner wirtschaftlichen Existenz (beispielsweise auch im Zusammenhang mit Unternehmen) gefährdet werden soll. ${ }^{57}$ Die Stundung erfolgt entweder aufgrund einer letztwilligen Verfügung des Verstorbenen oder auf Antrag des Pflichtteilsschuldners durch das Gericht.

Der letztwillig Verfügende kann die Stundung des Pflichtteilanspruchs gemäß $§ 766$ ABGB auf höchstens fünf Jahre nach seinem Tod anordnen. Weiters ist auch eine Ratenzahlung für diesen Zeitraum möglich. In besonders berücksichtigungswürdigen Fällen kann auch eine Verlängerung auf höchstens zehn Jahre durch das Gericht erfolgen. Der Pflichtteilsberechtigte ist an diese Anordnung grundsätzlich gebunden, es sei denn dies würde ihn unter Berücksichtigung aller Umstände unbillig hart treffen. Die Materialien zum ErbRÄG 2015 führen hierzu beispielhaft an, dass der Pflichtteilsberechtigte den Pflichtteil zur Sicherung seiner Existenz oder jener seiner nächsten Angehörigen dringend benötigt. $^{58}$

Wie bereits eingangs erwähnt, besteht auch für den Pflichtteilsschuldner die Möglichkeit, die Stundung bei Gericht zu beantragen, wenn ihn die Erfüllung des Pflichtteils unbillig hart träfe. Der Gesetzestext nennt beispielsweise jenen Fall, in dem der Pflichtteilsschuldner die Wohnung, die ihm zur Befriedigung seines dringenden Wohnbedürfnisses dient, oder ein Unternehmen, das seine wirtschaftliche Lebensgrundlage darstellt, veräußern müsste. Hierbei sind jedoch auch die Interessen der Pflichtteilsberechtigten angemessen $\mathrm{zu}$ berücksichtigen. Kommt es zur Stundung des Pflichtteilanspruchs, so fallen für diesen Zeitraum die gesetzlichen Zinsen in Höhe von 4\% pro Jahr an.

\subsection{Verjährung}

Das Recht, den Geldpflichtteil zu fordern, verjährt gemäß $\$ 1487$ a Absatz 1 ABGB in drei Jahren ab Kenntnis der für das Bestehen des Anspruchs maßgebenden Tatsachen. Unabhängig von dieser Kenntnis verjährt der Anspruch 30 Jahre nach dem Tod des Verstorbenen.

\subsection{Anrechnung von Schenkungen auf den Pflichtteil}

Wie bereits erwähnt, ist der Pflichtteil in Geld zu leisten; er kann jedoch durch eine Schenkung unter Lebenden oder eine Zuwendung von Todes wegen gedeckt werden. ${ }^{59}$ Wird dadurch nur ein Teil des Pflichtteils erfüllt, so steht dem Pflichtteilsberechtigten gemäß $\S 763$ ABGB ein Pflichtteilsergänzungsanspruch in Geld zu. Der Pflichtteilsberechtigte hat keinen Anspruch auf die Zuweisung bestimmter Sachen. Aus diesem Grund muss er sich letztwillige Zuwendungen gefallen lassen und kann diese somit nicht ausschlagen und stattdessen den Geldpflichtteil fordern. ${ }^{60}$ Weiters kann der Verstobene Zuwendungen auch mit Bedingungen und Belastungen hinterlassen, welche jedoch bei der Bewertung Berücksichtigung finden. ${ }^{61}$

Letztwillige Zuwendungen können beispielsweise als gesetzlicher Erbteil, Vermächtnis oder als Begünstigung aus einer vom Verstorbenen errichteten Privatstiftung erfolgen. ${ }^{62}$ Der Ehegatte muss sich zudem auch das Vorausvermächtnis des $\S 745$ ABGB anrechnen lassen. Die Anrechnung von Schenkungen unter Lebenden auf den Pflichtteil ist demgegenüber in den \$§ $781 \mathrm{ff} \mathrm{ABGB}$ geregelt und sieht vor, dass Schenkungen des Verstorbenen unter bestimmten Voraussetzungen der Verlassenschaft hinzuzurechnen sind und die Pflichtteile vom so erhöhten Verlassenschaftswert zu berechnen sind, was zu einer Erhöhung der Pflichtteile führt. ${ }^{63}$ Ist der Beschenkte selbst pflichtteilsberechtigt, muss er sich das Geschenk auf den (erhöhten) Pflichtteil anrechnen lassen, wenn ihm die Anrechnung nicht vom Verstorbenen erlassen worden ist. Finden die durch Hinzurechnung der Schenkungen erhöhten Pflichtteile in der Verlassenschaft keine Deckung, so können die verkürzten Pflichtteilsberechtigten unter bestimmten Voraussetzungen von den Beschenkten die Zahlung des fehlenden Betrags verlangen. ${ }^{64}$ Die Bestimmungen zur Hinzu- und Anrechnung von Schenkungen unter Lebenden sind sehr umfangreich und teils auch kompliziert ausgestaltet, weshalb die genaue Erörterung dieses Teilbereichs den Rahmen der Arbeit sprengen würde.

59. § $761 \mathrm{ABGB}$

60. Apathy, Erbrecht ${ }^{5}$ Rz 4/9; Perner/Spitzer/Kodek, Bürgerliches Recht ${ }^{5}$ (2016) 616; vgl. dazu § 808 Abs. 2 ABGB: 'Eine pflichtteilsberechtigte Person kann die Erbschaft nicht unter dem Vorbehalt ihres Pflichtteils ausschlagen.'

61. §762 ABGB

62. $\S 780 \mathrm{ABGB}$

63. Vgl. auch Eccher, Erbrecht ${ }^{6}$ Rz $12 / 4$.

64. $\S \S 789 \mathrm{ff}$ ABGB.
Das ist der gerichtliche Beschluss, durch den der Erbe nach Durchfüh $A B G B)$.

55. § 761 Abs. 1 ABGB. Siehe hierzu noch Kapitel 4.8.

56. § 778 Abs. 2 ABGB.

57. Eccher, Erbrecht ${ }^{6}$ Rz 12/37.

58. ErläutRV 688 BlgNR 25. GP 27 


\subsection{Pflichtteilsähnliche Rechte}

Ein pflichtteilsähnliches Recht stellt in Österreich das bereits in Kapitel 2.2 erörterte Vorausvermächtnis dar.

Durch das ErbRÄG 2015 wurde noch ein weiteres pflichtteilsähnliches Recht in die österreichische Rechtsordnung integriert; das sogenannte Pflegevermächtnis nach § 677 ABGB. Dieses steht dem Verstorbenen nahestehenden Personen (gesetzliche Erben und deren Ehegatte oder Lebensgefährte sowie deren Kinder; Lebensgefährte des Verstorbenen und dessen Kinder) zu, die den Verstorbenen in den letzten drei Jahren vor seinem Tod mindestens sechs Monate in nicht bloß geringfügigem Ausmaß (mindestens 20 Stunden im Monat) ${ }^{65}$ gepflegt haben. Dies gilt nur insoweit, als keine Zuwendung gewährt oder kein Entgelt vereinbart wurde. Unter Pflege wird dem Gesetzestext zufolge jede Tätigkeit verstanden, die dazu dient, einer pflegebedürftigen Person soweit wie möglich die notwendige Betreuung und Hilfe zu sichern sowie die Möglichkeit $\mathrm{zu}$ verbessern, ein selbstbestimmtes, bedürfnisorientiertes Leben zu führen. Somit muss es sich nicht um eine professionelle Krankenpflege handeln. ${ }^{66}$ Die Höhe des Vermächtnisses richtet sich nach Art, Umfang und Dauer der Leistungen. ${ }^{67}$ Sie orientiert sich insbesondere am Nutzen für die gepflegte Person, wie beispielsweise die Ersparnis von Aufwendungen für eine andere Pflegehilfe. ${ }^{68}$ Das Pflegevermächtnis gebührt neben dem Pflichtteil und kann nur bei Vorliegen eines Enterbungsgrundes entzogen werden. ${ }^{69}$ 